\title{
Clinical, Epidemiological, Biochemical Profile and Outcomes of Dengue and Dengue-like Illness in Children
}

\author{
Himani Dhingra ${ }^{1}$, Bhushan Kathuria ${ }^{2}$, Chandrahas T Deshmukh ${ }^{3}$
}

\begin{abstract}
Introduction: Dengue viral infection presents with a highly complex pathophysiological, economic, and ecologic problems. Through this study we aim to analyze the changing epidemiology and seasonal clustering of dengue fever cases. Also, we aim to analyze the clinical presentation, biochemical parameters, treatment given, and determine whether they have any association with the disease severity and outcome.

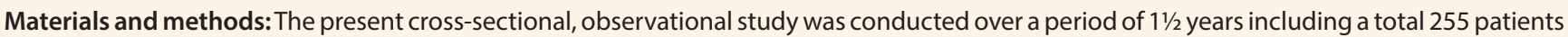
of probable dengue, confirmed dengue, and dengue-like illness (DLI). Statistical association of various qualitative and quantitative parameters of the disease was made with both disease severity and disease outcome.

Results: There were $27.8 \%$ patients in the age group $10-12$ years and $11 \%$ infants in our study. Maximum patients were admitted in the postmonsoon months. Fever was the most common symptom and abdominal tenderness was the most common sign. Leucopenia was the most frequent hematological abnormality noted in our study. Mean day of appearance of hematological derangements corroborated with the onset of critical phase of dengue. The NS1 antigen test was found to be most useful for diagnosis with maximum percentage positivity, rapid results, ease of availability, and cost-effectiveness. Mean day of illness for NS1 positivity was $4.6 \pm 2.9$ days. Ultrasound abdomen was a sensitive test to predict early signs of plasma leakage. Substantial number of patients could be managed with oral fluids only; however, a few required an intravenous (iv) crystalloid bolus at the time of presentation. Blood products were sparingly used in our study. Antibiotic use did not alter disease course and outcome, hence is not recommended. Very few patients needed intensive care unit (ICU) admission. There were 13 deaths (mortality rate of 5.1\%) during the study period. The rate of development of complications and also mortality was quite considerable in the DLI group. Hence, from the present study we emphasize that the DLI group should not be alienated from the continuum of spectrum of dengue viral infections. Conclusion: Presence of bleeding, encephalopathy, clinical/radiological signs of plasma leakage, hypotension, hepatomegaly, coagulopathy, deranged liver function test (LFT), and hemoconcentration with thrombocytopenia are indicative of a severe dengue disease and can also be used as surrogate markers for poor prognosis.
\end{abstract}

Keywords: Dengue fluid management, Dengue-like illness, Dengue in children, NS1 antigen.

Pediatric Infectious Disease (2020): 10.5005/jp-journals-10081-1235

\section{INTRODUCTION}

Dengue is the one of the most rapidly spreading mosquito-borne arboviral disease in the world. As per the National Vector Borne Diseases Control Program (NVBDCP-2013) data, Maharashtra has the highest dengue mortality rate (3.4\%) in India. Most patients recover following a self-limiting disease course. However, some can progress to develop severe disease, mostly characterized by plasma leakage with or without bleeding. Children often experience mild initial symptoms but are at a greater risk of developing severe complications. The diagnosis of acute dengue infection is mainly clinical. In dengue-endemic countries, cases of dengue infection should be notified early so that appropriate mosquito control measures can be initiated promptly.

Despite myriad of complex manifestations, management is relatively simple hydration therapy, which is very effective in reducing morbidity and mortality. Strict vitals monitoring with serial hematocrit $(\mathrm{Hct})$ testing along with a conservative intervention policy allow optimal titration of fluid therapy.

The most common causes of mortality in dengue are prolonged shock, massive hemorrhage, and fluid overload. The main problem leading to poor prognosis or death is not being diagnosed when presenting in critical conditions at the hospitals. ${ }^{1}$ We conducted the present study to explore of a set of preferably routine parameters that are easy to investigate and which could be used as indicators to
${ }^{1}$ Department of Pediatrics Oncology and Hematology, Indraprastha Apollo Hospitals, New Delhi, India

2Department of Otolaryngology, Head and Neck, Pandit Bhagwat Dayal Sharma Postgraduate Institute of Medical Sciences, Rohtak, Haryana, India

${ }^{3}$ Department of Pediatrics, King Edward Memorial Hospital and Seth Gordhandas Sunderdas Medical College, Mumbai, Maharashtra, India

Corresponding Author: Himani Dhingra, Department of Pediatrics Oncology and Hematology, Indraprastha Apollo Hospitals, New Delhi, India, Phone: +91 7015072317, e-mail: drhimani458@gmail.com

How to cite this article: Dhingra H, Kathuria B, Deshmukh CT. Clinical, Epidemiological, Biochemical Profile and Outcomes of Dengue and Dengue-like Illness in Children. Pediatr Inf Dis 2020;2(1):1-5.

Source of support: Nil

Conflict of interest: None

predict the severity of dengue infection especially in the pediatric age group.

Primary prevention of dengue is currently possible only with personal protection from mosquito bites and overall improving vector control. However, the development of vaccines and drugs has the potential to change this. The high index of suspicion based on clinical signs and symptoms, timely laboratories diagnosis of

() The Author(s). 2020 Open Access This article is distributed under the terms of the Creative Commons Attribution 4.0 International License (https://creativecommons. org/licenses/by-nc/4.0/), which permits unrestricted use, distribution, and non-commercial reproduction in any medium, provided you give appropriate credit to the original author(s) and the source, provide a link to the Creative Commons license, and indicate if changes were made. The Creative Commons Public Domain Dedication waiver (http://creativecommons.org/publicdomain/zero/1.0/) applies to the data made available in this article, unless otherwise stated. 
dengue coupled with prompt supportive management, general awareness among the public, and constant vigilance by the healthcare officials could help in combating the morbidity and mortality associate with dengue.

\section{Materials and Methods}

This study was a cross-sectional, observational study conducted over a period of $1 \frac{1}{2}$ years (Feb 2014-Oct 2015) in the department of pediatrics at a tertiary care center in Maharashtra, India. Standard WHO 2009 definitions were used for classifying dengue cases as clinical, probable, confirmed, and dengue-like illness (DLI). All children aged between 1 month and 12 years of age, admitted with clinical dengue, during the study period were included. All the three lab tests, i.e., NS1 antigen, IgM serology, and dengue PCR were done for all the patients, irrespective of the day of illness. Either of the three tests positive was enrolled as a probable or confirmed dengue. However, patients admitted with all the three tests negative were enrolled as DLI. These patients were not tested for dengue lgG. Although, other common monsoon illnesses like malaria, leptospirosis, and other common causes of pediatric febrile illnesses were ruled out in the DLI group. This group was examined parallel to the other three severity groups of dengue. The enrolment of the study participants was done after a written informed consent of the parents. Children beyond the study age group and/or not consenting for participation were excluded. The data was recorded in a predesigned case record form.

\section{Statistical Analysis}

Taking into consideration the previous year's admission rate for dengue and DLI to our department, the estimated sample size of 200 was proposed. The statistical association of various clinical and laboratory parameters was studied in the different disease severity groups and also in subgroup of DLI. Similar association was also analyzed with disease outcomes in the form of recovery and mortality.

The statistical analysis of the data included descriptive analysis and difference between groups was analyzed by Chi-square tests or Mann-Whitney $U$ tests. Univariate and multivariate regression analyzes were used to evaluate the relationship between prognostic indicators and disease severity. All $p$ values $<0.05$ were considered as significant.

\section{Results and Observation}

In total, 255 patients were included in the study. Tables 1 and 2 show the age and genderwise distribution of patients, respectively. During our study, maximum patients were admitted in the month of September. A rise in number of dengue cases was seen in postmonsoon months. Most of the patients recovered with routine care and management; however, 36 (14.1\%) patients required admission in ICU. Mean number of days of hospital stay were 4.5, with minimum duration being 3 hours to maximum being 22 days. Mean number of days of ICU stay were $4.5 \pm 3.4$. The most common indication for ICU admission was shock.

Varied symptomatology was noted at the time of presentation to the hospital. The most common symptom being fever (100\%) followed by nausea/vomiting. Mean day of appearance of rash was $4 \pm 2.4$ days. In patients presenting with bleeding manifestations, the gastrointestinal tract (GIT) was the most common site of bleed (45\%) followed by skin bleeds (26\%). Major life-threatening bleeds
Table 1: Agewise distribution of patients

\begin{tabular}{lcc}
\hline Age (months) & No. & Percentage \\
\hline$<24$ & 33 & 12.9 \\
$24-47$ & 17 & 6.7 \\
$48-71$ & 40 & 15.7 \\
$72-95$ & 46 & 18.0 \\
$96-119$ & 48 & 18.8 \\
$120-144$ & 71 & 27.8 \\
Total & 255 & 100.0 \\
\hline
\end{tabular}

Table 2: Genderwise distribution of patients

\begin{tabular}{lll}
\hline Gender & No. & Percentage \\
\hline Female & 123 & 48.2 \\
Male & 132 & 51.8 \\
\hline
\end{tabular}

Table 3: Frequency of various symptoms at the time of presentation

\begin{tabular}{lc}
\hline Symptom & Percentage $(n=255)$ \\
\hline Fever & $100(255)$ \\
Nausea/vomiting & $60(153)$ \\
Malaise & $48.2(123)$ \\
Rash & $37.6(96)$ \\
Headache & $29.4(75)$ \\
Musculoskeletal pain & $24.7(63)$ \\
Bleeding & $16.5(42)$ \\
Oliguria & $9.4(24)$ \\
Seizures & $7.1(18)$ \\
Retro-orbital pain & $5.1(13)$ \\
Altered sensorium & $4.3(11)$ \\
Jaundice & $0.8(02)$ \\
\hline
\end{tabular}

were quite rare. Abdominal tenderness was the most common sign noted. Tables 3 and 4 summarize the frequency of symptoms and signs, respectively, at the time of presentation.

Leucopenia was the most frequent hematological abnormality noted (Table 5). Minimum and maximum total leukocyte count (TLC) as noted were 600 and $20,600 / \mathrm{mm}^{3}$, respectively, with a mean TLC of $4776.48 / \mathrm{mm}^{3}$. Mean day of lowest TLC was $5.4 \pm 3.1$. Highest mean hemoglobin $(\mathrm{Hb})$ was $12.7 \pm 1.9$ and highest mean Hct was $37.9 \pm 6.2$. Mean day of hemoconcentration was $4.9 \pm 2.9$. Mean platelet count was $109994.9 \pm 78886.6$. Mean day of lowest platelet count was $5.2 \pm 3.14$. Mean platelet count was lowest among the severe dengue group. The chronology of appearance of hematological abnormalities corroborated with onset of critical phase, which is usually after 4-5 days of onset of fever. Out of 72 patients (28\%) who had raised transaminases, 4 patients showed significant transaminitis (>1000 IU/mL) and features of fulminant hepatic failure (FHF). The blood gas analysis was not available for all the patients; however, among the 61 available results 30 patients showed abnormality and most common abnormality noted was metabolic acidosis. In our study, ascites was a more common sign of plasma leakage than pleural effusion. The frequency of radiological abnormalities is summarized in Table 6.

The distribution of patients in various disease severity groups and the DLI group is shown in Figure 1. Specific dengue diagnostic 
Clinical, Epidemiological, Biochemical Profile and Outcomes of Dengue and Dengue-like Illness in Children

Table 4: Frequency of various signs at the time of presentation

\begin{tabular}{ll}
\hline Findings & Percentage $(n=255)$ \\
\hline Abdominal tenderness & $46.3(118)$ \\
Hepatomegaly & $35.3(90)$ \\
Conjunctival suffusion & $35.3(90)$ \\
Hypotension & $31.8(81)$ \\
Third space loss & $16.5(42)$ \\
Pharyngeal congestion & $8.6(22)$ \\
Splenomegaly & $5.5(14)$ \\
\hline
\end{tabular}

Table 5: Frequency of laboratory derangements in patients

\begin{tabular}{lc}
\hline Parameters & Percentage $(n=255)$ \\
\hline Leukopenia & $63.5(162)$ \\
Hemoconcentration & $63.1(161)$ \\
Thrombocytopenia & $56.1(143)$ \\
Deranged liver function tests & $28(72)$ \\
Deranged coagulation profile & $6.6(17)$ \\
Deranged renal function tests & $0.8(02)$ \\
\hline
\end{tabular}

Table 6: Radiological abnormalities in patients

\begin{tabular}{ll}
\hline Parameters & Percentage $(n=255)$ \\
\hline Ascites & $26.7(68)$ \\
$\begin{array}{l}\text { Pseudothickening of gallbladder } \\
\text { wall }\end{array}$ & $24.7(63)$ \\
Pleural effusion & $18.8(48)$ \\
\hline
\end{tabular}

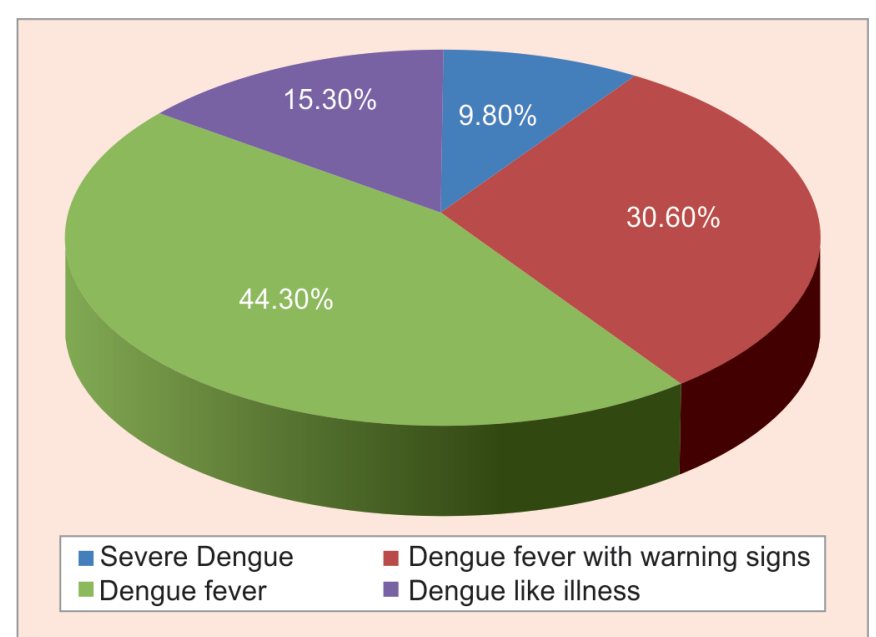

Fig. 1: Patient distribution based on disease severity at the time of presentation

tests were performed on all the patients. Of the 216 patients of confirmed dengue (either of the three tests positive), maximum percentage positivity (87.5\%) was for the NS1 antigen followed by PCR (percentage positivity $51.8 \%$ ) and least for $\lg M(24.1 \%)$. Mean day of illness for positivity of the diagnostic tests was almost similar for NS1 and IgM $(4.6 \pm 2.9)$ and was slightly later for PCR $(5.3 \pm 2.9)$.

Significant number of patients (45.9\%) were managed with oral fluids only; iv fluid resuscitation was required (54.1\%) for the rest of the patients. In our study, 81 patients had hypotension on
Table 7: Frequency of various supportive measures used in management

\begin{tabular}{ll}
\hline Intervention & Percentage $(n=255)$ \\
\hline Crystalloid bolus & $30.6(78)$ \\
Antibiotics & $27.1(69)$ \\
Inotropes & $14.5(37)$ \\
Colloid transfusion & $7.5(19)$ \\
Ventilation & $5.1(13)$ \\
\hline
\end{tabular}

Table 8: List of complications

\begin{tabular}{lc}
\hline Complication & Percentage $(n=40)$ \\
\hline Shock & $90(36)$ \\
Meningoencephalitis & $17.5(7)$ \\
Acute liver failure & $12.5(5)$ \\
Acute respiratory distress & $10(4)$ \\
Fluid overload & $5(2)$ \\
Acute renal failure & $2.5(1)$ \\
Secondary bacterial infection & $2.5(1)$ \\
\hline
\end{tabular}

presentation of which 78 patients required a crystalloid bolus for resuscitation. Inotropes were more frequently used than colloid transfusion (7.5\%) to treat shock in our study (Table 7). Transfusion requirement was minimal in our cohort. Less than $10 \%$ patients required any form of blood products. In total, 40 patients (15.7\%) developed complications, which are enlisted in Table 8.

Most of the patients (94.9\%) recovered without any residual morbidity and were discharged. There were 13 deaths during our study period with the percentage mortality of $5.1 \%$. Most of the deaths (46.2\%) were in the severe dengue group followed by $30.8 \%$ deaths in the DLI group. Not all patients in this group had a mild disease. Around $10 \%$ patients required intensive care admission and significant percentage of patients also required crystalloid bolus, colloid infusion, and inotropes. Early clinical suspicion and appropriate management akin to the severe dengue group can reduce mortality in this group.

\section{Association of Various Parameters with Disease Severity and Outcomes}

As stated by the WHO 2009 criteria, the warning signs are indicative of impending severe dengue. In concordance with this, bleeding, radiological signs of plasma leakage, hypotension, hepatomegaly, and hemoconcentration with thrombocytopenia showed statistically significant association with disease severity and outcome (all $p$ values $<0.05$ ). Though nausea/vomiting was the second most common symptom in our cohort, no significant association was seen with either disease severity $(p$ value $=0.612)$ or outcome ( $p$ value $=1)$. Presence of rash ( $p$ value $=0.023$ ) and/or abdominal tenderness ( $p$ value $=0.0003$ ) at the time of presentation was usually associated with severe disease. However, their presence did not always predict a negative outcome ( $p$ value $=0.247,0.151$, respectively). Leucopenia, though very common lab derangement noted in dengue, did not show any significant association with disease severity ( $p$ value $=0.06$ ) or mortality $(p$ value $=1)$. Though rare, but neurological manifestations like encephalopathy $\left(p\right.$ value $\left.=9.5 \times 10^{-6}\right)$, coagulopathy $\left(p\right.$ value $\left.=2.1 \times 10^{-9}\right)$, and transaminitis ( $p$ value $=4.4 \times 10^{-8}$ ) indicated a more severe disease and hence increased mortality. 
Percentage survival improved with administration of crystalloid bolus. Our study revealed that colloid transfusion did not alter the disease course and was rather associated with poor outcome among patients ( $p$ value $<0.001$ ). Empiric antibiotic therapy was used in critically ill patients, suspecting secondary bacterial infection. Percentage survival in the group that did not receive antibiotics was $100 \%$ indicating good prognosis. Since, antibiotic usage did not alter the disease course and outcome. This indicates that there is no rationale of using antibiotics in dengue.

We compared a list of quantitative variables of our study among the two outcome groups (recovery and mortality) using the Mann-Whitney Wilcoxon test. There was a significant difference of distribution in the mean platelet count, which was much lower in the mortality group in comparison to the recovery ( $p$ value $=$ 0.00052). Similarly, there was longer hospitalization and longer ICU stay in the mortality group ( $p$ value $=0.004$ and 0.002 , respectively).

\section{Discussion}

Once a neglected tropical disease, dengue is now a major international public health concern. There is no specific treatment for severe dengue, but early detection and access to proper medical care lowers fatality rates below $1 \%$.

In our study, maximum percentage of patients were in the age group of 10-12 years. Mean age of presentation was 6.7 years. Dengue viral infection is usually more common in children of the older age group due to more outdoor activity and increased mosquito contact. Similar mean age of presentation has also been reported in previous studies ${ }^{2,3}$ from India. However, our study also revealed significant number of patients in the age group of less than 2 years. Of these, $11 \%$ were infants; the youngest patient in our study was 1 month old. Quite a few infants presented with severe dengue, presenting with similar severity like a secondary dengue infection seen in older patients. This could be attributed to the passively transferred circulating maternal IgG antibodies, due to dengue endemicity. Another hypothesis to explain these cases of dengue infection in earlier age groups could be due to increased virulence of virus irrespective of the pre-infection antibody status. In a hospital-based study from Nicargua, Hammond et al. ${ }^{4}$ have reported increased burden of severe dengue in infants and children 5-9 years of age.

Our study showed clustering of dengue cases during months of July to October. This corresponds to the post-monsoon season in our region as mosquito breeding is maximum during this period. Similar findings have also been reported in previous studies from Mumbai ${ }^{2}$ and northeastern India. ${ }^{5}$ This characteristic epidemiological factor of seasonal clustering can help arrange better preparedness of hospitals and improved implication of vector control programs.

Dengue infection usually presents as an acute febrile illness with nonspecific constellation of symptoms without any obvious focus of infection. Most of the previous studies ${ }^{3,5}$ have reported similar symptomatology as noted in our study. The constitutional symptoms are usually higher in dengue as compared to other febrile illnesses; this is a useful clinical guide in endemic areas. As early clinical diagnosis and prompt fluid management are the two main highlights to avoid negative outcomes.

Neurological manifestations are atypical presentations in dengue fever. In our study also, few patients with severe disease presented with seizures and encephalopathy. Previous studies by Aggarwal et al. ${ }^{6}$ and Pawaria et al. ${ }^{7}$ have reported encephalopathy as an atypical presenting feature of dengue infection. Though rare, the incidence of neurological manifestations in dengue has been reported to be between $0.5 \%$ and $6.2 \%$. As febrile seizure is a commoner differential in children, dengue encephalopathy should always be considered in a given clinical scenario.

Not all patients of dengue with thrombocytopenia had clinical bleeding. In our study, no correlation was observed between mean platelet count and frequency of bleeding manifestations. Similar finding has also been observed by Joshi et al. ${ }^{8}$ in their study. In another study by Lum et al. ${ }^{9}$ duration of shock and low-normal $\mathrm{Hct}$ at the time of shock have been considered as risk factors of hemorrhage in severe dengue. In this study too, platelet count alone did not predict bleeding.

Dengue viral infection can have a wide range of hepatic manifestations ranging from asymptomatic transaminitis to FHF. In the present study, transaminitis was more commonly seen in association with severe disease and was associated with higher mortality especially in patients who developed FHF. Jagadishkumar et al. ${ }^{10}$ have described the spectrum of hepatic manifestations in children with dengue. They concluded that hepatic dysfunction was more common in the dengue shock syndrome group and there was no correlation between degree of hepatomegaly and LFT derangement.

Most of the previously published studies ${ }^{8,10}$ have used dengue $\operatorname{lgM}$ as the diagnostic modality. The PCR-based literature is not yet readily available. From our study, the dengue NS1antigen test showed the highest positivity rate and was the earliest test to be positive (in terms of days of illness). Also being a rapid diagnostic test and least expensive of all, this test was found to be an ideal specific diagnostic test to aid in early diagnosis of dengue viral infections and thus help in managing patients accordingly.

Most of the dengue patients in our study were managed in ward with routine care; only $14 \%$ patients required admission in ICU with most common indication being persistent hypotension. In a previous study from south India, ${ }^{11}$ persistent shock and need for ventilatory support were the two most common indications for ICU admission.

Management in dengue is predominantly supportive and includes judicious fluid therapy in order to prevent or treat shock while at the same time preventing fluid overload due to capillary leak. Crystalloids were preferred over colloids for initial resuscitation. Inotropes and colloids were sparingly used in our study. Similar principles of choice of fluid therapy have been reported in previous studies., 92,13

Platelet transfusions were used very sparingly in our study. Only critically ill patients with platelet count $<20,000 / \mathrm{mm}^{3}$ with evidence of clinical bleeding and/or prolonged shock were given platelet transfusion. However, this did not change the disease course or decrease bleeding and was ultimately associated with poor outcome in such patients. Similar observations have been reported by Khan Assir et al. ${ }^{14}$ in a randomized controlled trial in adult patients where platelet transfusion neither prevented development of severe bleeding nor shortened time to cessation of bleeding in dengue viral infections. Sharma et al. ${ }^{15}$ have also emphasized for a conservative approach to platelet transfusions in dengue.

Fresh frozen plasma (FFP) transfusion, colloid infusion, inotropes, and antibiotics were more frequently used in critically ill patients with severe disease. However, no significant alteration was noted in the disease course with the use of any of these agents. Statistically significant association was proved between 
use of these agents and mortality. Not many studies have previously analyzed the risk benefit ratio of using antibiotics and FFP transfusion in pediatric dengue infections.

The case fatality rate of our study was comparable to previous study by Kamath et al. ${ }^{11}$ However, this was slightly higher than most of the other previous studies, ${ }^{2,8,12}$ which can be attributed to delay in seeking medical attention and limited ICU facilities.

In our study, presence of bleeding, hypotension, hepatomegaly, coagulopathy, deranged LFT, and signs of plasma leakage were early predictors of mortality. However, presence of rash, nausea/ vomiting, and abdominal tenderness showed no significant association with mortality. In a previous Brazilian study, Branco Mdos et al. ${ }^{16}$ found epistaxis and persistent vomiting was associated with death in children with severe dengue. Laoprasopwattana et al. ${ }^{17}$ concluded that in patients with severe dengue, overt bleeding and acute respiratory failure are two important mortality predictors. In the meta-analysis done by Zhang et al. ${ }^{18}$ symptoms and signs like gastrointestinal bleeding (hematemesis/malena), nausea/vomiting, skin rash, hepatomegaly and abdominal pain were predictive of development of severe dengue disease.

\section{Conclusion}

In cases with suspected dengue infection, simple, readily available, and cost-effective test in the form of complete blood count can aid in management especially in resource-constraint settings. The USG abdomen constitutes an important investigation for detecting plasma leakage, which is missed clinically and hence can be used as an early predictor of disease severity before overt clinical manifestations appear. Emphasis should be laid on early aggressive fluid therapy. Presence of bleeding, hypotension, hepatomegaly, encephalopathy, coagulopathy, deranged LFT, and signs of plasma leakage were early predictors of mortality.

\section{Limitations of the Study}

All the serial cases admitted with dengue infection could not be included in the study due to logistic constraints; this could have led to selection bias. Primary and secondary infections were not differentiated with laboratory evidence. Paired sera testing for demonstration of fourfold rise in antibody titer against dengue virus was not done due to lack of follow-up of all patients. Dengue virus isolation and typing for the commonest circulating strain was not done during the study as the test was not available in house. Dengue IgG tests were not performed for any patients, not even in the DLI group.

\section{References}

1. Pongpan S, Wisitwong A, Tawichasri C, et al. Prognostic indicators for dengue infection severity. Int J Clin Pediatr 2013;2(1):12-18. DOI: 10.4021/ijcp73w.
2. Shah I, Deshpande GC, Tardeja PN. Outbreaks of dengue in Mumbai and predictive markers of dengue shock syndrome. J Trop Pediatr 2004;50(5):301-305. DOI: 10.1093/tropej/50.5.301.

3. Kulkarni JM, Sarathi V, Bhalla V, et al. Clinico-epidemiological profile of children hospitalized with dengue. Indian J Pediatr 2010;77(10): 1103-1107. DOI: 10.1007/s12098-010-0202-2.

4. Hammond SN, Balmaseda A, Pérez $L$, et al. Differences in dengue severity in infants, children, and adults in a 3-year hospital-based study in Nicaragua. Am J Trop Med Hyg 2005;73(6):1063-1070. DOI: 10.4269/ajtmh.2005.73.1063.

5. Dutta P, Khan S, Borah J, et al. Demographic and clinical features of patients with dengue in northeastern region of India: a retrospective cross-sectional study during 2009-2011. Journal of Virology \& Microbiology 2012;2012:1-11. DOI: 10.5171/2012.786298.

6. Aggarwal A, Kumar P, Faridi MM. Neurological manifestation as presenting feature of dengue infection. J Pediatr Neurosci 2015;10(1):76-77. DOI: 10.4103/1817-1745.154362.

7. Pawaria A, Mishra D, Juneja M, et al. Atypical manifestations of dengue fever. Indian Pediatr 2014;51(6):495-496. DOI: 10.1007/s13312-0140434-8.

8. Joshi R, Baid V. Profile of dengue patients admitted to a tertiary care hospital in Mumbai. Turk J Pediatr 2011;53(6):626-631.

9. Lum LCS, Goh AYT, Chan PWK, et al. Risk factors for hemorrhage in severe dengue infections. J Pediatr 2002;140(5):629-631. DOI: 10.1067/ mpd.2002.123665.

10. Jagadishkumar K, Jain P, Manjunath VG, et al. Hepatic involvement in dengue fever in children. Iran J Pediatr 2012;22(2):231-236.

11. Kamath S, Ranjit S. Clinical features, complications and atypical manifestations of children with severe forms of dengue hemorrhagic fever in South India. Indian J Pediatr 2006;73(10):889-895. DOI: 10.1007/BF02859281.

12. Bhave S, Rajput CS, Bhave S. Clinical profile and outcome of dengue fever and dengue haemorrhagic fever in paediatric age group with special reference to who guidelines (2012) on fluid management of dengue fever. 2015;3(4):196-201.

13. Wills BA, Nguyen MD, Ha TL, et al. Comparison of three fluid solutions for resuscitation in dengue shock syndrome. N Engl J Med 2005;353(9):877-889. DOI: 10.1056/NEJMoa044057.

14. Khan Assir MZ, Kamran U, Ahmad HI, et al. Effectiveness of platelet transfusion in dengue fever: a randomized controlled trial. Transfus Med Hemother 2013;40(5):362-368. DOI: 10.1159/000354837.

15. Sharma A, Charles K, Chadee D, et al. Dengue hemorrhagic fever in Trinidad and Tobago: a case for a conservative approach to platelet transfusion. Am J Trop Med Hyg 2012;86(3):531-535. DOI: 10.4269/ ajtmh.2012.10-0209.

16. Branco Mdos R, Luna EJ, Braga Júnior LL, et al. Risk factors associated with death in Brazilian children with severe dengue: a casecontrol study. Clinics (Sao Paulo) 2014;69(1):55-60. DOI: 10.6061/ clinics/2014(01)08.

17. Laoprasopwattana K, Chaimongkol W, Pruekprasert P, et al. Acute respiratory failure and active bleeding are the important fatality predictive factors for severe dengue viral infection. PLoS ONE 2014;9(12):e114499. DOI: 10.1371/journal.pone.0114499.

18. Zhang $H$, Zhou $Y P$, Peng $H J$, et al. Predictive symptoms and signs of severe dengue disease for patients with dengue fever: a metaanalysis. Biomed Res Int 2014;2014:359308. DOI: 10.1155/2014/ 359308. 